\title{
The role of immature granulocyte percentage in predicting acute chest syndrome and the severity of the vaso-occlusive crisis in sickle cell disease
}

\author{
Feryal Karahan ${ }^{1 \oplus}$, Selma Ünal ${ }^{1 \oplus}$, Dilara Bal Topçu ${ }^{2 \oplus}$, Yeşim Öztaş ${ }^{3 \oplus}$, Gülçin Bozlư ${ }^{4 \oplus}$ \\ ${ }^{1}$ Department of Pediatric Hematology, Mersin University Faculty of Medicine, Mersin; ${ }^{2}$ Department of Medical Biochemistry, Akyurt \\ State Hospital, Ankara; ${ }^{3}$ Department of Medical Biochemistry, Faculty of Medicine, Hacettepe University, Ankara; ${ }^{4}$ Department of \\ Pediatric Emergency Medicine, Mersin University Faculty of Medicine, Mersin, Turkey.
}

\begin{abstract}
Background. Sickle cell disease (SCD) is an inflammatory disease that can result in both chronic and acute inflammation. Immature granulocytes (IG) are not-yet-mature white blood cells that can be easily detected in complete blood count (CBC) tests. In recent studies it has been suggested that IG may play a role in determining the prognosis of inflammatory diseases. The aim of our study was to investigate the role of IG percentage on predicting acute chest syndrome (ACS) and the severity of vaso-occlusive crisis (VOC) in patients with SCD.

Methods. The study cohort consisted of 49 SCD patients admitted to the emergency department for VOC. If symptoms did not regress despite appropriate treatment including hydration and analgesia, they were hospitalized. Patients whose symptoms regressed were discharged from the emergency department within 24 hours. Blood samples, including CBC and C-reactive protein (CRP), a marker of inflammation, were taken within the first hour of admission. Steady state laboratory parameters from the previous visit in the last three months were collected from patient files.

Results. The mean age was $18 \pm 4$ (range 8-25) years. Most were hospitalized $(41 / 49 ; 83.7 \%)$ and 8 of 49 were discharged from the emergency department after their treatment for VOC. ACS developed in 13 of 49 (26.5\%). White blood cell, neutrophil and nucleated red blood cell counts, percentage of IG (IG\%) and CRP levels were significantly increased in patients with VOC. IG\% of patients with ACS was significantly higher than patients without ACS. However, ROC analysis showed that IG\% was not associated with the development of ACS or hospitalization for VOC.

Conclusions. Despite a small SCD cohort, the significant increase in the IG\% in patients with VOC compared to their baseline values has suggested a role for IG\% in predicting VOC. Although IG\% was higher in ACS, its utility in predicting ACS was poor.
\end{abstract}

Key words: sickle cell disease, inflammation, immature granulocyte.

Sickle cell disease (SCD) arises due to a point mutation in the gene encoding the beta globin subunit. Hypoxia, acidosis and dehydration induce the polymerization of sickle hemoglobin $(\mathrm{HbS})$ that results in decreased deformability of red blood cells (RBC). This results in vasoocclusive crisis (VOC), ischemia-reperfusion injury, and endothelial dysfunction. ${ }^{1}$

$凶$ Feryal Karahan

drferyalkarahan@gmail.com

Received 11th May 2021, revised 4th August 2021, accepted 10th September 2021.
Sickle cell vasculopathy is characterized by sterile inflammation and neutrophil counts are higher in SCD patients, both when the disease is stable, the steady-state, and during exacerbation such as VOC, than in healthy controls. It has been reported that high neutrophil counts were associated with acute chest syndrome (ACS), silent brain infarcts, hemorrhagic strokes, and early death in SCD patients., In SCD, neutrophils have increased adhesive properties and adhesion of neutrophils to the inflammation-induced endothelium and to 
sickle erythrocytes is particularly important in VOC pathogenesis. ${ }^{3-5}$

Immature granulocytes (IG) are a not-yetmature subset of white blood cells that can be easily detected in complete blood count tests. IG contain granules in their cytoplasm and include metamyelocytes, myelocytes and promyelocytes. The IG count is an indicator of bone marrow activation, particularly leukopoiesis, and raised IG counts are found in response to inflammatory conditions, as well as in the neonatal period or in pregnancy. Recent studies have suggested a role for the IG percentage (\%) in peripheral blood in determining the prognosis of inflammatory diseases. ${ }^{6-10}$

While the role of neutrophils in the pathogenesis of VOC is known, there is no data about the relation of IG\% with VOC. The aim of this study was to investigate IG\% in the steady state and during VOC in SCD, and to evaluate its potential role in predicting VOC. We also aimed to determine the relationship between IG\% and other inflammatory parameters in SCD.

\section{Material and Methods}

This retrospective study included SCD patients that were under follow-up between November 2020 and February 2021 by the department of Pediatric Hematology and were admitted to the emergency department for VOC. If the symptoms did not regress, despite appropriate treatments such as hydration and analgesics, they were hospitalized. Patients whose symptoms regressed were discharged from the emergency department within 24 hours. The patients who had another inflammatory disease and infection were excluded from this study. ACS was diagnosed by the presence of fever and/or respiratory symptoms and a new pulmonary infiltrate on chest X-Ray.

Blood samples were taken from all patients within the first hour of admission. Complete blood count (CBC) was performed on an automated hematology analyzer, XN-1000
(Sysmex Corp., Kobe, JAPAN). IG\% was calculated from the white cell differential channel based on granularity and nucleic acid content. Steady state laboratory parameters from the previous visit and within the three months prior to the emergency admission were collected from patient records.

The study was approved by Mersin University Ethical Board (number: 03.03.2021; 2021/220).

Statistical analyses were performed using SPSS Statistics for Windows, version 20 (SPSS Inc., Chicago, IL, USA). Continuous variables were expressed as mean \pm standard deviation (SD) while frequency and percentage (\%) were used to define categorical data. Categorical variables were analyzed using the Chi-square test and Fischer's exact test. Normality of distribution was examined using the Kolmogorov-Smirnov test. Mann-Whitney $U$ test was used for comparisons between two groups.

Receiver Operating Curve (ROC) analysis was performed to determine the effect of IG\% on having ACS or hospitalization for VOC. The areas under the curve (AUC) of the IG\% parameter, statistical significance level, cutoff sensitivity and specificity values were calculated.

\section{Results}

During the study period a total of 49 SCD patients (43 HbSS and $6 \mathrm{HbSß}$ ) were admitted to the emergency department for VOC. The mean $\pm S D$ age was $18 \pm 4$ (range 8 -25) years. Thirty-two patients $(67 \%)$ were male and 17 (33\%) were female. Twenty-three patients had ACS, 15 patients had avascular necrosis (AVN) and three patients had a history of stroke. The mean annual VOC frequency of the patients was $1.7 \pm 0.88$ (range 1-5). Eleven patients (22\%) were transfused and three patients were on the chronic transfusion program in the last year. Of the 49 patients, $46(93.9 \%)$ were on hydroxyurea treatment.

Forty-one of $49 \quad(83.7 \%)$ patients were hospitalized and the remaining eight were 
discharged from the emergency department after their treatment for VOC. ACS developed in 13 of $49(26.5 \%)$ patients.

All inflammatory markers, including leucocyte (WBC) and neutrophil counts, C-reactive protein (CRP) levels, and IG\%, were significantly different during VOC compared with the previous steady state values (Table I). Platelet counts and hemoglobin $(\mathrm{Hb})$ concentrations were significantly decreased and WBC and neutrophil counts, nucleated red blood cell (NRBC) \%, IG\% and CRP levels were significantly increased in VOC.

$\mathrm{Hb}$ levels were significantly lower $(\mathrm{p}=0.027)$ and CRP levels were significantly higher $(p=0.030)$ in patients who were hospitalized compared to patients discharged from the emergency department (Table II). There was no correlation between IG\% and length of stay in the patients who were hospitalized.

The IG\% of patients with ACS was significantly higher than patients without ACS (Table III). Using ROC analysis to investigate the relationship of IG\% and ACS, the cut-off value for IG\% was $>1 \%$, specificity $60.53 \%$, sensitivity $57.14 \%$, and the AUC was 0.52 . Thus IG\% was not discriminatory for ACS ( $\mathrm{p}=0.82)$. Similarly IG\% was not significant in identifying which patients would be hospitalized for VOC ( $p=0.829$; specificity $41.7 \%$; sensitivity $75 \%$; and AUC $=0.524)$.

When SCD patients were grouped according to genotype, IG\% was significantly higher in SS than SB patients during VOC $(\mathrm{p}=0.016)$. All

Table I. Laboratory parameters of the patients $(n=49)$ during steady state and VOC.

\begin{tabular}{lccc}
\hline & Steady state & VOC & p value \\
\hline WBC $((\mu \mathrm{L})$ & $11.261 \pm 3.391$ & $17.215 \pm 5967$ & 0.001 \\
$\mathrm{Hb}(\mathrm{g} / \mathrm{dL})$ & $9.3 \pm 1.1$ & $9.0 \pm 1.5$ & 0.037 \\
Platelet $(\mu \mathrm{L})$ & $471.204 \pm 192.504$ & $387.122 \pm 198.561$ & 0.006 \\
Neutrophil $(\mu \mathrm{L})$ & $5.302 \pm 1.952$ & $9.431 \pm 4.083$ & 0.001 \\
$\mathrm{IG} \%$ & $0.37 \pm 0.18$ & $1.35 \pm 1.46$ & 0.001 \\
$\mathrm{NRBC} \%$ & $1.38 \pm 2.37$ & $3.59 \pm 7.62$ & 0.017 \\
$\mathrm{MPV}(\mathrm{fl})$ & $8.17 \pm 3.45$ & $8.42 \pm 3.26$ & 0.374 \\
$\mathrm{CRP}(\mathrm{mg} / \mathrm{dL})$ & $12.32 \pm 33.00$ & $14.85 \pm 15.90$ & 0.001 \\
\hline
\end{tabular}

VOC: Vaso-occlusive crisis, WBC: White blood cell, Hb: Hemoglobin, IG\%: immature granulocyte percentage, NRBC:

Nucleated red blood cell, MPV: Mean platelet volume, CRP: C-reactive protein

Table II. Comparison of laboratory parameters in the SCD patients who were hospitalized or non hospitalized for VOC.

\begin{tabular}{lccc}
\hline & $\begin{array}{c}\text { Non-hospitalized patients } \\
(\mathrm{n}=8)\end{array}$ & $\begin{array}{c}\text { Hospitalized patients } \\
(\mathrm{n}=41)\end{array}$ & P value \\
\hline $\mathrm{WBC}(\mu \mathrm{L})$ & $18.030 \pm 3.947$ & $17.055 \pm 6.098$ & 0.438 \\
$\mathrm{Hb}(\mathrm{g} / \mathrm{dL})$ & $9.9 \pm 1.0$ & $8.8 \pm 1.6$ & 0.027 \\
Platelet $(\mu \mathrm{L})$ & $463.500 \pm 138.558$ & $372.220 \pm 202.294$ & 0.193 \\
Neutrophil $(\mu \mathrm{L})$ & $9.219 \pm 4.963$ & $9.472 \pm 3.961$ & 0.895 \\
$\mathrm{IG} \%$ & $1.20 \pm 0.86$ & $1.38 \pm 1.60$ & 0.559 \\
$\mathrm{NRBC} \%$ & $1.57 \pm 1.20$ & $4.02 \pm 8.47$ & 0.391 \\
$\mathrm{MPV}(\mathrm{fl})$ & $8.23 \pm 3.36$ & $8.46 \pm 3.28$ & 0.523 \\
$\mathrm{CRP}(\mathrm{mg} / \mathrm{dL})$ & $8.42 \pm 10.64$ & $16.10 \pm 10.64$ & 0.030 \\
\hline
\end{tabular}

SCD: sickle cell disease, VOC: vaso-occlusive crisis, WBC: white blood cell, Hb: hemoglobin, IG\%: immature granulocyte percentage, NRBC: nucleated red blood cell, MPV: mean platelet volume, CRP: C-reactive protein 
Table III. SCD patients with or without acute chest syndrome (ACS).

\begin{tabular}{lccc}
\hline & ACS $(\mathrm{n}=13)$ & Without ACS $(\mathrm{n}=36)$ & $\mathrm{p}$ value \\
\hline WBC $(\mu \mathrm{L})$ & $17.816 \pm 8.532$ & $16.998 \pm 8.532$ & 0.526 \\
$\mathrm{Hb}(\mathrm{g} / \mathrm{dL})$ & $9.4 \pm 2.0$ & $8.8 \pm 1.3$ & 0.196 \\
Platelet $(\mu \mathrm{L})$ & $365.838 \pm 235.301$ & $394.808 \pm 186.704$ & 0.571 \\
Neutrophil $(\mu \mathrm{L})$ & $8.562 \pm 3.659$ & $9.744 \pm 4.230$ & 0.428 \\
IG\% & $2.48 \pm 2.18$ & $1.00 \pm 0.8$ & 0.009 \\
NRBC $\%$ & $2.95 \pm 7.0$ & $3.86 \pm 8.15$ & 0.545 \\
MPV (fl) & $8.22 \pm 3.71$ & $8.49 \pm 3.13$ & 0.856 \\
CRP $(\mathrm{mg} / \mathrm{dL})$ & $15.39 \pm 12.78$ & $14.65 \pm 17.0$ & 0.556 \\
\hline
\end{tabular}

SCD: sickle cell disease, ACS: acute chest syndrome, WBC: white blood cell, Hb: hemoglobin, IG\%: immature granulocyte percentage, NRBC: nucleated red blood cell, MPV: mean platelet volume, CRP: C-reactive protein

other $\mathrm{CBC}$ and inflammatory parameters were similar. There was no correlation between IG\% and the history of the type of sickle cell disease, AVN, ACS, stroke and annual frequency of painful crisis. In addition, no correlation was found between chronic transfusion and IG\%.

\section{Discussion}

Immature granulocytes are produced and differentiated in bone marrow, and their presence in the circulation indicates greatly increased bone marrow activation due to infectious or inflammatory conditions. ${ }^{11}$ Additionally, IG\% may be elevated in other conditions, like cancer and during pregnancy. To our knowledge, the relationship of IG\% with clinical and laboratory findings in SCD has not been investigated to date. In our study, IG\% level was found to be significantly higher during VOC than when the disease is stable, the steady state. Similarly, patients with ACS had significantly higher IG\% than the patients without ACS.

CRP is recognized as a good marker of acute and chronic inflammation and is frequently used in SCD patients. High levels of CRP at steady state are associated with an increased frequency of acute pain and increased levels during VOC have been reported to be valuable in predicting the development of ACS in SCD patients. ${ }^{12}$ We found high CRP levels in patients with SCD both during steady state and VOC. CRP levels were significantly higher in the patients who were hospitalized than the patients discharged from the emergency department after their treatment for $\operatorname{VOC}(\mathrm{p}=0.03)$.

It is known that neutrophil counts are high in patients with SCD, both during steady state and VOC, and is also related with increased mortality risk. ${ }^{3}$ In our study, both WBC and neutrophil counts were increased during VOC compared to steady state levels $(p<0.001)$. However, there was no difference in $\mathrm{WBC}$ and neutrophil counts between patients with VOC who were hospitalized and those discharged from the emergency department. In addition there was no correlation between WBC and neutrophil counts were not determinants of the length of hospital stay.

IG level was used as an early biomarker to show infection and inflammatory status in some studies. IG\% was found to be increased in a study of patients with peripheral enthesitis and correlated with CRP elevation and clinical activity. ${ }^{13} \mathrm{Narcl}$ et al. ${ }^{8}$ investigated $\mathrm{IG} \%$ in patients with upper gastrointestinal bleeding and reported that IG\% was significantly higher in patients who died compared with patients who were discharged. The IG\% was specific $(93.8 \%)$ and sensitive $(100 \%)$ in predicting in-hospital mortality. Unal et al. ${ }^{9}$ suggested that increased IG\% is a simple, fast, and effective marker in the early prediction of acute necrotizing pancreatitis. Additionally, Güngör et al. $^{10}$ reported that IG\% had higher 
sensitivity and specificity in predicting systemic inflammatory response syndrome in patients with acute pancreatitis. However, Park et al. ${ }^{7}$ suggested that the diagnostic ability of IG\% was insufficient in patients with acute and complicated appendicitis, and was of no additional benefit in investigating appendicitis compared with other inflammatory markers.

In our study, IG \% was found to be significantly higher during VOC than the steady state $(p<0.001)$. However, there was no difference in IG\% between the patients who were hospitalized and the patients discharged from the emergency department. In addition, there was no correlation between the IG\% and length of stay in the patients who were hospitalized. Also, ROC analysis indicated that IG\% was not discriminatory for hospitalization for VOC.

ACS is an important cause of morbidity and mortality in children and adults with SCD. Several risk factors have been associated with the development of ACS, such as young age, low $\mathrm{HbF}$, high baseline hemoglobin, steadystate leukocytosis, and airway hypersensitivity. Since two-thirds of ACS episodes occur in patients hospitalized for VOC, identifying biomarkers to predict ACS occurrence would be of clinical benefit. ${ }^{14}$ In our study, ACS developed in $26.5 \%$ of patients who were admitted to the emergency department because of VOC. The IG\% of patients with ACS was significantly higher than patients without ACS. Despite this, ROC analysis showed that the IG\% parameter did not have discriminatory power for the occurrence of ACS.

Biomarker investigations are ongoing to predict VOC in SCD. While the role of IG\%, an easily calculated parameter in $\mathrm{CBC}$, has been investigated in determining the severity of diseases in many infectious or inflammatory diseases, it has been evaluated for the first time in SCD in this study. Despite the limited number of patients, the significant increase in IG\% in patients with VOC compared to the steady state has suggested a role for IG\% in predicting VOC in SCD. Although IG\% was higher in patients with ACS, analysis showed that this was insufficiently discriminatory in predicting ACS.

\section{Ethical approval}

The study was approved by Mersin University Ethical Board (number: 03.03.2021; 2021/220).

\section{Author contribution}

All authors confirm their contribution with data input and interpretation, drafting the manuscript. FK; draft manuscript preparation. SÜ; study conception and design. DBT; analysis of biochemical data. YÖ; analysis and interpretation of results. GB; data collection. All authors reviewed the results and approved the final version of the manuscript.

\section{Source of funding}

The authors declare the study received no funding.

\section{Conflict of interest}

The authors declare that there is no conflict of interest.

\section{REFERENCES}

1. Shah F, Dwidedi M. Pathophysiology and recent therapeutic insights of sickle cell disease. Ann Hematol 2020; 99: 925-935. https://doi.org/10.1007/ s00277-020-03977-9

2. Zhang D, Xu C, Manwani D, Frenette PS Neutrophils, platelets, and inflammatory pathways at the nexus of sickle cell disease pathophysiology. Blood 2016; 18: 801-809. https://doi.org/10.1182/ blood-2015-09-618538

3. Okpala I, Daniel Y, Haynes R, Odoemene D, Goldman J. Relationship between the clinical manifestations of sickle cell disease and the expression of adhesion molecules on white blood cells. Eur J Haematol 2002; 69: 135-144. https://doi.org/10.1034/j.16000609.2002.02775.x 
4. Turhan A, Weiss LA, Mohandas N, Coller BS, Frenette PS. Primary role for adherent leukocytes in sickle cell vascular occlusion: a new paradigm. Proc Natl Acad Sci 2002; 99: 3047-3051. https://doi. org/10.1073/pnas.052522799

5. Bennewitz MF, Jimenez MA, Vats R, et al. Lung vasoocclusion in sickle cell disease mediated by arteriolar neutrophil-platelet microemboli. JCI insight 2017; 2: 1-18. https://doi.org/10.1172/jci.insight.89761

6. Lipiński M, Rydzewska G. Immature granulocytes predict severe acute pancreatitis independently of systemic inflammatory response syndrome. Prz Gastroenterol 2017; 12: 140-144. https://doi. org/10.5114/pg.2017.68116

7. Park JS, Kim JS, Kim YJ, Kim WY. Utility of the immature granulocyte percentage for diagnosing acute appendicitis among clinically suspected appendicitis in adult. J Clin Lab Anal 2018; 32: 1-89. https://doi.org/10.1002/jcla.22458

8. Narcı H, Berkeşoğlu M, Üçbilek E, Ayrık C. The usefulness of the percentage of immature granulocytes in predicting in-hospital mortality in patients with upper gastrointestinal bleeding. Am J Emerg Med 2021; 46: 646-650. https://doi. org/10.1016/j.ajem.2020.12.039

9. Ünal $\mathrm{Y}$, Barlas AM. Role of increased immature granulocyte percentage in the early prediction of acute necrotizing pancreatitis. Ulus Travma Acil Cerrahi Derg 2019; 25: 177-182. https://doi. org/10.14744/tjtes.2019.70679
10. Güngör A, Göktuğ A, Tekeli A, et al. Evaluation of the accuracy of immature granulocyte percentage in predicting pediatric serious bacterial infection. Int J Lab Hematol 2021; 43: 632-637. https://doi. org/10.1111/ijlh.13474

11. Nierhaus A, Klatte S, Linssen J, Eismann NM, et al. Revisiting the white blood cell count: immature granulocytes count as a diagnostic marker to discriminate between SIRS and sepsis - a prospective, observational study. BMC Immunology 2013; 14: 1-8. https://doi.org/10.1186/1471-2172-14-8

12. Krishnan S, Setty Y, Betal SG, et al. Increased levels of the inflammatory biomarker C-reactive protein at baseline are associated with childhood sickle cell vasocclusive crises. Br J Haematol 2010; 148: 797-804. https://doi.org/10.1111/j.1365-2141.2009.08013.x

13. Al-Ansari A, Hussein I. Immature granulocytes levels is a potential biomrker in peripheral enthesitis. Ann Rheum Dis 2019; 78: 1533. https:// doi.org/10.1136/annrheumdis-2019-eular.4348

14. Kalpatthi R, Novelli EM. Measuring success: utility of biomarkers in sickle cell disease clinical trials and care. Hematology Am Soc Hematol Educ Program 2018; 30: 482-492. https://doi.org/10.1182/ asheducation-2018.1.482 